\title{
A Case Of Atypical Gastric Carcinoma With Osteoclast Like Giant Cells
}

\author{
Rahul Pandit*, Irina A. Danilova
}

\begin{abstract}
Out of all the different types of neoplasms affecting the stomach, gastric carcinomas with Osteoclast-like Giant Cells (OGC) is one of the most uncommon. Although OGC are typically found in osseous neoplasms and tumors of the tendon sheath, few cases of extra-skeletal neoplasms with OGC have been documented. These typically involve organs such as the pancreas, gall-bladder, kidney, and breast. Even though the role of OGC in histogenesis of such tumors still remains unclear, their presence in extra-osseous neoplasms may indicate a certain level of immune reaction of the host towards the neoplastic transformation of normal tissue. We report a case of a 70-year-old Caucasian female hospitalized for evaluation of epigastric pain. Further examinations including endoscopy and biopsy of the stomach revealed gastric adenocarcinoma with OGC. This report also provides a brief insight into the possible immune reaction in such neoplasms
\end{abstract}

\section{KEYWORDS: Osteoclast like Giant Cells (OGC), adeno-carcinoma}

\section{INTRODUCTION}

Osteoclast-Like Giant Cells (OGC) are multinucleated large cells with clear cytoplasm and are encountered in relatively uncommon tumors of the bone tissue and the tendon sheath. Although these osseous tumors are generally benign by nature and have a slow growth, they still have a recurrence rate of over $50 \%$ and may give pulmonary metastasis.

The presence of OGC in different extra-skeletal neoplasms has been cited in various literatures $[1,2,3,4]$ with the involvement of organs including the skin, breast, pancreas, gall-bladder and the kidneys. Though the origin of these cells has been proposed to be of the mononuclear-macrophage lineage, their actual role in the histogenesis in these extra-osseous tumors is ill-defined.

In this case report we present a detailed case of a patient with gastric adeno-carcinoma with OGC. A special focus will be laid on the immune reaction in the tumor tissue of such neoplasms with a brief

*To whom correspondence should be addressed:

Rahul Pandit

Piskarevsky Prospect 47/23

Saint Petersburg, Russia, 195067

Telephone:+7(812)543-93-24

Fax:+7(812)543-93-24

Email:onlyrahu115@yahoo.com discussion on the differential diagnosis of such neoplasms with anaplastic carcinomas which also manifest with atypical giant nucleated cells.

\section{CASE REPORT}

A 70-year-old Caucasian female was admitted to the Surgical Department of the St. Petersburg State Medical Academy with complaints of epigastric pain in May 2004. Detailed history revealed a 6-month history of nausea, epigastrial pain and progressive generalized weakness. A soft abdomen with slight epigastrial tenderness was found on palpation. Laboratory investigations showed anemia, hypoproteinemia and dysproteinemia. A fibro-oesophago-gastro-duodenoscopy revealed a stomach with relatively rigid walls. A polypoid protrusion of the gastric mucosa along with multiple erosions with a tendency to bleed in the region of the gastric cardia and the small curvature was noticed. Of the three biopsy specimens, the one obtained from the esophagus displayed signs of inflammation whereas the ones from the erosions depicted definite malignant changes corresponding to gastric adeno-carcinoma. Following diagnosis, a gastrectomy with D1 lymph node dissection and oesophago-jejunostomy with an inter-intestinal anastomosis was performed. The surgical TNM was finalized as ST3N2M0. 


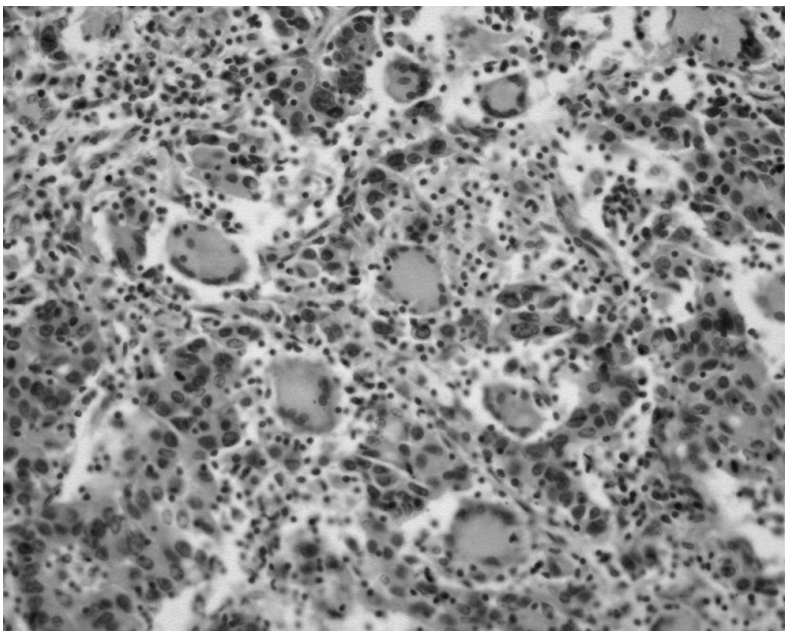

Figure 1. Moderately differentiated malignant glands with prominent lympho-histocytic infiltration of the stroma. Magnification 100X.

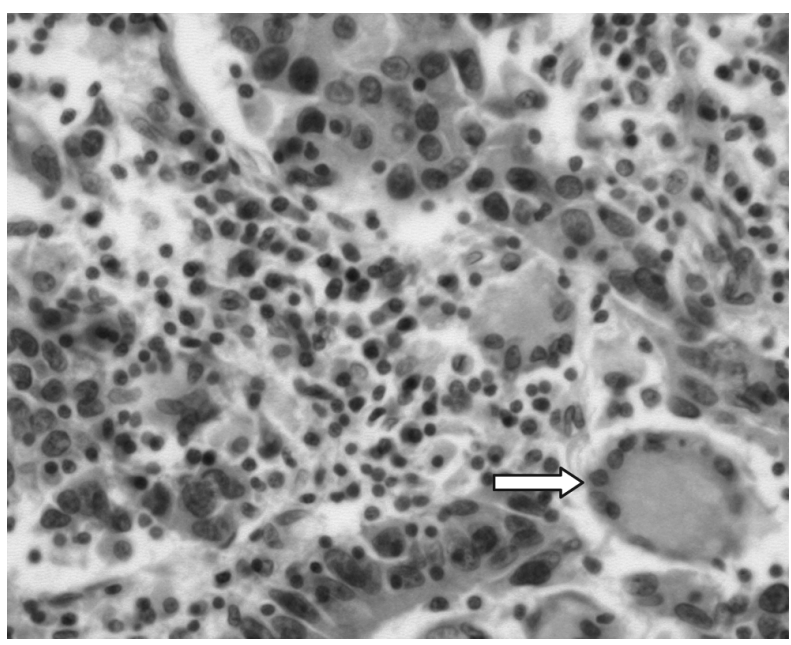

Figure 2. Arrow indicating stromal localization of a typical giant cell with multiple nuclei distributed circumferentially. Magnification 400X.

\section{MACROSCOPIC FINDINGS}

A tumor involving the gastric cardia and small curvature was presented as a polyp like mass with distinct ulcerative changes and blood clots on its surface. Noted dimensions were $10 \times 10 \times 6 \mathrm{~cm}$. The cut surface of the tumor revealed a whitish-pink mass that had an invasive pattern of growth and involved all the layers of the stomach wall but without any encroachment into the surrounding organs. The tumor was classified as Bor 2 according to Bormann's scale.

\section{MICROSCOPIC FINDINGS}

The tumor was diagnosed as being a low differentiated adeno-carcinoma with specific stromal infiltration accompanying multinucleated giant cells and extensive lympho-histiocytic infiltration (Figure

\begin{tabular}{|l|l|l|l|}
\hline \hline Antibodies & Source & Staining & Dilution \\
\hline \hline CD 8 & $*$ & Membrane & RTU \\
\hline CD 4 & $*$ & Membrane & RTU \\
\hline CD68 & $*$ & Cytoplasmic & $1: 40$ \\
\hline PCK & $*$ & Cytoplasmic & RTU \\
\hline PCNA & $* *$ & Nuclear & $1: 200$ \\
\hline BCL2 & $* *$ & Cytoplasmic & $1: 50$ \\
\hline EBV & $*$ & Cytoplasmic & $1: 100$ \\
\hline Ki 67 & $* *$ & Nuclear & \\
\hline \hline
\end{tabular}

Table 1. List of primary monoclonal antibodies used in immunohistochemistry. PCK-Pan- cytokeratin, EBV-Epstein Barr Virus, PCNA- Proliferating Cell Nuclear Antigen, *Novocastra England, **DAKO,Germany RTU- Ready to Use antibodies

1 and Figure 2). The giant cells were predominantly located in the stroma around the malignant glands with seldom peri-glandular localization. The giant cells were characterized by a clear cytoplasm and multiple nuclei ranging between 3 to 7 , arranged either circumferentially or randomly in the cytoplasm. Eight of the lymph nodes isolated from the region of the small curvature of the stomach were positive for metastases. The margins of gastrectomy presented with normal gastric tissue, thus confirming the success of the operation. The pTNM was inferred as $\mathrm{pT} 3 \mathrm{~N} 2 \mathrm{M} 0$.

\section{IMMUNOHISTOCHEMISTRY}

Staining for different immunohistochemical markers, listed in Table 1 were carried out on $6 \mu \mathrm{m}$ thick tissue samples placed on polyvinyl slides, with appropriate positive and negative controls. The Labeled Streptavidin Biotin (LSAB-DAKO) detection system was used for detection of primary antibodies. Staining with Pan- cytokeratin (Figure 4) was strongly positive for the poorly differentiated malignant gastric glands without any staining of the intervening stromal compartment. In contrast, the stromal compartment was highly stained by the CD68 marker, whereas the staining in the malignant epithelium was almost negligible (Figure 3). Interestingly, staining with CD4 and CD8 revealed mostly stromal infiltration by the latter and dual stromal and glandular infiltration by the former.

Note that the tumor tissue, and the regional lymph nodes, were positively stained for the latent nuclear antigen of EBV. Ki67 immunostaining surprisingly showed total absence of any proliferation in the tumor tissue sample, while the anti-apoptotic marker, BCL 2, was only moderately expressed.

\section{CONCLUSION}

Giant cell carcinomas of the stomach belong to a 


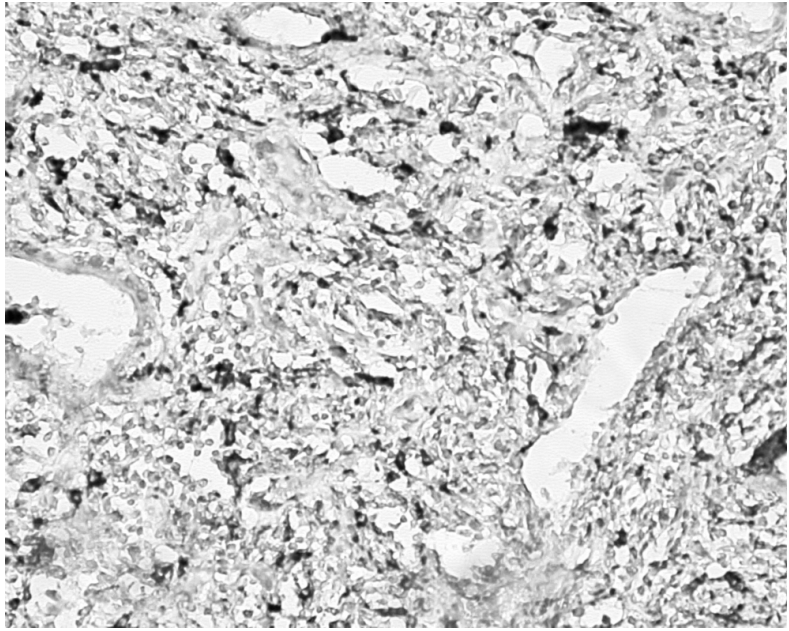

Figure 3. CD68 staining showing the positive staining of stromal elements with relatively free glands. Magnification 200X.

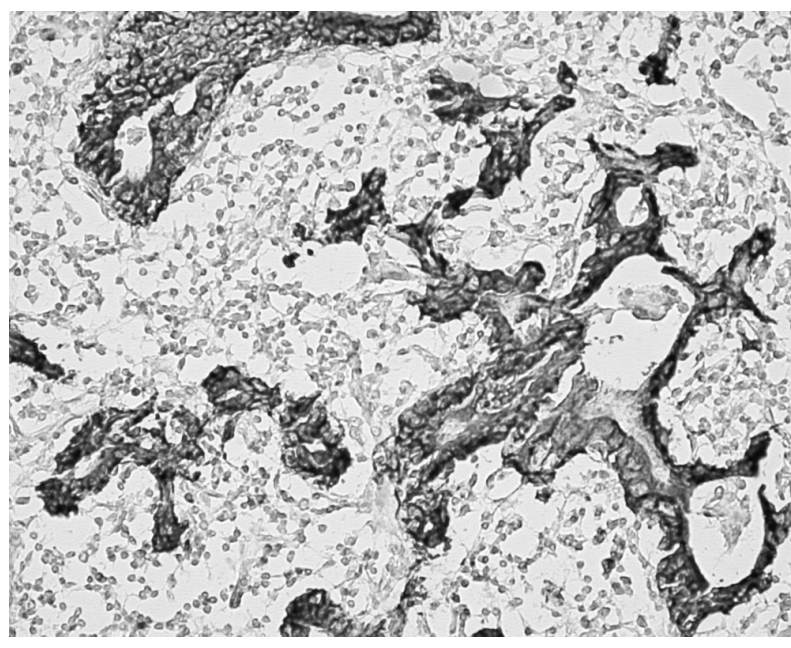

Figure 4. Positive staining of malignant glands for PanCytokeratin antibodies. Magnification 200X.

group of rare neoplasms. These neoplasms are of epithelial origin and were confirmed by positive pancytokeratin staining. Given the intense immune reaction in these tumors, and presence of multinuclear giant cells, a crucial question remains as to whether such neoplasms are more aggressive than other adenocarcinomas without such elements.

Adeno-carcinomas of the stomach with OGC have to be also carefully differentiated from anaplastic carcinomas, which are usually characterized by faster growth, early metastasis and a much poorer prognosis. Anaplastic cancer cells may present with several morphological features and may have from a single to multiple nuclei but in all cases the giant cells will be of epithelial origin and hence will stain negative to CD68 or other mesenchymal markers thus validating their epithelial nature.

Although the etiology of the lymphocytic infiltration of the tumors is not entirely known, EBV infection has been argued to be a probable cause and has been isolated in various cases of lymphoepitheliomalike tumors (LELT) $[5,6]$. These tumors, although typical for the nasopharynx, have also been encountered in the stomach, bladder and intestinal mucosa, and are identifiable by lympho-histiocytic infiltration. Furthermore, such tumors have also been cited to have a better prognosis in comparison to GC cases without EBV infection [7].

The prognostic index in this case was proposed by running immunohistochemical tests on prominent independent markers, Ki67 and Bcl2. Researchers have diverse opinions on the prognostic value of $\mathrm{Bcl} 2$ expression in gastric cancer $[8,9,10,11,12]$. While traditional view connects the over-expression of $\mathrm{Bcl} 2$ to low tumor apoptotic ability and hence a poorer prognosis, studies have also linked the expression of $\mathrm{Bcl} 2$ as an indicator for biological activity of tumor [13]. In our case, we see mild expression of $\mathrm{Bcl} 2$ and negative expression of Ki67, thus suggesting a low biological activity of the tumor i.e. higher index of apoptosis and lower proliferative index. These results must be, however, considered in the light of the advanced TNM staging, which remains the best predictor in terms of survival.

To conclude, OGC represent uncommon tumors of the stomach. The rarity with which such neoplasms present in clinical practice makes it difficult to make universal conclusions about the prognosis. They must, however, be distinguished from anaplastic carcinomas of the stomach, with a more diffuse pattern of growth and a poorer prognosis overall.

\section{ACKNOWLEDGMENTS:}

We would like to thank Dr. Irina Kostochek, D.O. Ott Research Institute of Obstetrics and Gynecology, St. Petersburg, Russia for her help and guidance with our immunohistochemical studies.

\section{REFERENCES:}

1. Young-E J et all -A Case of Osteoclast-like Giant Cell Tumor of the Pancreas with Ductal Adenocarcinoma: Histopathological, Immunohistochemical, Ultrastructural and Molecular Biological Studies-J Korean Med Sci 2005; 20: 516-20 ISSN 1011-8934

2. Shoichi D et all - Undifferentiated Spindle and Giant Cell Carcinoma of the Common Bile Duct- Tokai J Exp Clin Med., Vol. 28, No. 3, pp. 127-130, 2003

3. Baschinsky DY, Frankel WL, Niemann TH-Gastric carcinoma with osteoclast-like giant cells-Am J Gastroenterol. 1999 Jun; 94(6):1678-81

4. Stracca-Pansa V et all- Gastric carcinoma with osteoclast-like giant cells : report of four cases- $\mathrm{Am}$ So of $\mathrm{Cl}$ Patholo and the College of Am Patholo. Spring meeting, Orlando FL , (22/04/1995) 1995 , vol. 103 , no 4 , pp. 500-528 
5. Mayer, E. K , Beckley I. and Winkler M.H,Lymphoepithelioma-like carcinoma of the urinary bladderdiagnostic and clinical implications- Nature clinical practice urology (2007) 4 pp 197-171

6. Torlakovic G, Snover D. C., and Torlakovic E. -Simultaneous EBV-Positive Lymphoepithelioma-like Carcinoma and EBVNegative Intestinal-Type Adenocarcinoma in a Patient With Helicobacter pylori-Associated Chronic Gastritis- Am J Clin Pathol 2004

7. Shibata D, Tokunaga M, Uemura Y, Sato E, Tanaka S and Weiss L M- Association of Epstein-Barr virus with undifferentiated gastric carcinomas with intense lymphoid infiltration. Am J of Pathol, Vol 139, 469-474

8. Müller W, Schneiders A, Hommel G, Gabbert HE.-Prognostic value of BCL2 in gastric cancer- Anticancer research 1998 NovDec;18(6B):4699-704.

9. Zafirellis K, Karameris A, Milingos N, Androulakis G.Molecular markers in gastric cancer: can p53 and bcl-2 protein expressions be used as prognostic factors? Anticancer Research 2005 Sep-Oct;25(5):3629-36.

10. Forones NM, Carvalho AP, Giannotti-Filho O, Lourenço LG, Oshima CT.- Cell proliferation and apoptosis in gastric cancer and intestinal metaplasia.- Arq Gastroenterol. 2005 JanMar;42(1):30-4. Epub 2005 Jun 22

11. Ishii H, Gobé G, Kawakubo Y, Sato Y, Ebihara Y.Interrelationship between Epstein-Barr virus infection in gastric carcinomas and the expression of apoptosis-associated proteins.- Histopathology. $2001 \mathrm{Feb}$;38(2):111-9

12. Chang MS, Lee HS, Jung EJ, Kim CW, Lee BL, Kim WH.- Role and prognostic significance of proapoptotic proteins in EpsteinBarr virus-infected gastric carcinomas-Anticancer Research 2007 Mar-Apr;27(2):785-9

13. Kume T, Oshima K, Shinohara T, Takeo H, Yamashita Y, Shirakusa T, Kikuchi M.- Low rate of apoptosis and overexpression of bcl-2 in Epstein-Barr virus-associated gastric carcinoma.-Histopathology-1999 Jun;34(6):502-9

Rahul Pandit (MD 2008) is a graduate of I.I. Mechnikov St. Petersburg State Medical Academy and a prospective Masters student at Utrecht University (September 2008). His research interests include tumor morphology and microenvironment.

Irina A. Danilova ( MD 1984, PhD 2001) is a Docent (Associate Professor) and pathologist at the Department of Pathological Anatomy, I.I. Mechnikov St. Petersburg State Medical Academy. She is also the Vice-Dean and a member of the Scientific Medical Council at the St. Petersburg State Medical Academy Named After I.I. Mechnikov. Her research woks are directed towards tumor morphology, histiogenesis, microenvironment and epidemiological studies. 